\title{
The potential for $\alpha$ induced nuclear scattering, reaction and decay, and a resonance-pole-decay model with exact explicit analytical solutions
}

\author{
Basudeb Sahu ${ }^{1}$ and Swagatika Bhoi ${ }^{2}$ \\ 1 Department of Physics, College of Engineering \\ and Technology, Bhubaneswar-751003, India and \\ ${ }^{2}$ School of Physics, Sambalpur University, Jyoti Vihar, Burla, 768019, India
}

\begin{abstract}
The decay of $\alpha$ particle from a nucleus is viewed as a quantum resonance state of a two-body scattering process of the $\alpha+$ daughter nucleus pair governed by a novel nucleus-nucleus potential in squared Woods-Saxon form. By the application of the rigorous optical model (OM) potential scattering (S-matrix) theory the genuineness of the potential for the system is established by giving good explanation of the elastic scattering and reaction cross sections data of the $\alpha+$ nucleus pair. From the pole position in the complex momentum $(\mathrm{k})$ plane of the S-matrix defined above, the energy and width of the resonance state akin to the decaying state of emission of $\alpha$ particle are extracted and from this width, the result of $\alpha$-decay half-life is derived to account for the experimental result of half-life in the cases of large number of $\alpha$-emitters including heavy and super-heavy nuclei. The S-matrix of the full OM calculation above is replaced by an analytical function expressed in terms of exact Schrödinger solutions of a global potential that closely represents the Coulomb-nuclear interaction in the interior and the pure Coulomb wave functions outside, and the resonant poles of this S-matrix in the complex momentum plane are used to give satisfactory results of decay half-lives of $\alpha$ coming out from varieties of nuclei.
\end{abstract}

PACS number(s): 23.60.+e, 21.10.Tg, 23.70.+j, 27.90.+b

PACS numbers: 


\section{INTRODUCTION}

The process of decay of alpha $(\alpha)$ particle from heavy and super-heavy nuclei has been studied intensively in the past few years [1 12]. In many papers a simple two-body model was applied [11 13] and in most papers a potential was derived that was able to fit the measured $\alpha$-decay half-lives of the alpha emitters. In recent time, such a potential for the $\alpha+$ nucleus two-body interaction is generated microscopically in the double folding $(t \rho \rho$ approximation) using explicitly the nuclear (both proton and neutron) densities [4, 14]. However, most of the studies did not attempt to use these potentials for the description of other experimental quantities such as, for example, $\alpha$-scattering cross sections or reaction (fusion) cross sections. Using the potential extracted from the fitting of decay rate data, Devisov and Ikezoe [3] estimated the values of fusion cross section as a function of energy by treating fusion process as a one-dimensional barrier passing mechanism. Bhagwat and Gambhir [15] have tried to account for the measured results of fusion cross sections in some cases of $\alpha+$ nucleus systems by the similar one-dimensional treatment of the fusion process and found no success in explaining the data of fusion cross sections and the decay half-lives by using the potential obtained within the framework of mean field theory. In both the studies stated above, the potential under question has not been used or tested for the analysis of angular variation of the experimental values of differential scattering cross sections at different incident energies. It is well known that the genuineness of a nucleusnucleus potential rests on the satisfactory explanation of the above elastic scattering data in the optical model potential (OMP) analysis. Through this analysis only, can one know the exact height and radial position of the Coulomb-nuclear potential barrier. Using a potential, without proper verification of its barrier height and position, in the studies of other processes namely fusion and decay does not go well with the physical understanding of the processes.

In principle, the application of semi-classical model for tunneling is not necessary for the calculation of $\alpha$-decay half-lives and the fusion reaction cross section in the quantum mechanical two-body collision process of $\alpha$-nucleus system. From the potential, using the rigorous S-matrix (SM) theory of potential scattering, one can directly obtain the energy and decay width from the poles of the SM in the lower half of the complex momentum $(\mathrm{k})$ plane

close to the real axis [10]. Further, this SM method can be amalgamated within a code developed for calculating phase-shifts and cross sections in the same $\alpha+$ nucleus collision 
problem to explain the elastic scattering and reaction (fusion) cross section data in a unified

way [16, 17]. The motivation of this paper is to present a phenomenological potential for the $\alpha+$ nucleus system which is consistent with the potential generated by relativistic mean field (RMF) theory [18] and is suitable for simultaneous description of three important events of $\alpha$ induced nuclear reaction namely (i) elastic scattering, (ii) reaction, and (iii) $\alpha$ emission by giving satisfactory explanation of the measured quantities: elastic scattering cross section, reaction cross section and $\alpha$-decay half-life by the calculated results obtained using the SM theory of potential scattering.

Further, the combined Coulomb-nuclear potential adopted above is closely reproduced by a r-dependent potential expression. Using this potential form, we exactly solve the Schrödinger equation and match them with the analytical Coulomb wave functions outside and obtain an expression for the S-matrix explicitly as a function of the incident energy and the potential parameters. Then, from the pole position in the complex momentum $(\mathrm{k})$ plane of the S-matrix, we extract the energy and width of the resonance state akin to the decaying state of emission of $\alpha$ particle from a nucleus. With this simple model of potential scattering calculation we achieve good explanation of the experimental results of $\alpha$-decay half-lives in the cases of several $\alpha$-emitters that include heavy and super-heavy nuclei.

In Sec. II, the details of the OMP calculation and the derivation of the expression for the S-matrix of the exactly solvable potential are given. Section III discusses the applications of the formulation to the explanation of the experimental data of elastic scattering cross section, reaction cross section and $\alpha$-decay half-life. In Sec. IV, we present the summary and conclusion of the work.

\section{THEORETICAL FORMULATION}

The nuclear optical potential model is developed for the analysis of the results of scattering and reaction cross sections obtained in the measurements of nucleus-nucleus collisions. In this quantum collision theory, the following reduced radial Schrödinger equation

$$
\frac{d^{2} \phi(r)}{d r^{2}}+\frac{2 \mu}{\hbar^{2}}(E-V(r)) \phi(r)=0
$$


for a complex Coulomb-nuclear potential

$$
V(r)=V_{N}(r)+V_{C}(r)+V_{\ell}(r)
$$

the sum of the complex nuclear potential $\left(V_{N}(r)\right)$, the electrostatic potential $\left(V_{C}(r)\right)$, and the centrifugal potential $\left(V_{\ell}(r)\right)$ in the spatial region $0<r \leq R_{\max }$, a distance where the attractive nuclear potential becomes zero, is solved using the Runge-Kutta (RK) type of numerical integration or multistep potential approximation [19] and the wave function $(\phi(r))$ and its derivative $(d \phi(r) / d r)$ at the radial position $\mathrm{r}=R_{\max }$ are obtained.

In the outer region $r>R_{\max }$, the potential of the $\alpha+$ nucleus interaction is only Coulombic, $\mathrm{V}_{C}(r)$, with the centrifugal term $\mathrm{V}_{\ell}=\frac{\hbar^{2}}{2 \mu} \frac{\ell(\ell+1)}{r^{2}}$ for different angular momentum partial wave $\ell$. Here, $\mu$ stands for the reduced mass of the two-body system. Using the exact Coulomb wave functions, i.e., $\mathrm{F}_{\ell}(r)$ (regular) and $\mathrm{G}_{\ell}(r)$ (irregular) and their derivatives $\mathrm{F}_{\ell}^{\prime}(r)$ and $\mathrm{G}_{\ell}^{\prime}(r)$ in the outer region $r>R_{\max }$ and the wave function $\phi(r)$ and its derivative $\frac{d \phi(r)}{d r}$ in the left side of $r=R_{\max }$ and matching them at $r=R_{\max }$, we get the expression for the partial wave $\mathrm{S}$-matrix denoted by $\mathrm{S}_{\ell}$ as

$$
S_{\ell}=2 i C_{\ell}+1
$$

where

$$
\begin{gathered}
C_{\ell}=\frac{k F_{\ell}^{\prime}-F_{\ell} H}{H\left(G_{\ell}+i F_{\ell}\right)-k\left(G_{\ell}^{\prime}+i F_{\ell}^{\prime}\right)}, \\
H=\left.\frac{d \phi / d r}{\phi}\right|_{r=R_{\max }} .
\end{gathered}
$$

with $k=\sqrt{\frac{2 \mu}{\hbar^{2}} E}$ for the incident energy E. In (4), prime $\left({ }^{\prime}\right)$ denotes derivative with respect to $\rho=\mathrm{kr}$.

We estimate the results of differential elastic scattering cross section in ratio to Rutherford scattering as a function of scattering angle $(\theta)$ by using the $\mathrm{S}$-matrix, $\mathrm{S}_{\ell}$, given by (3) in the following expression:

$$
\frac{d \sigma_{e l}}{d \sigma_{R}}=\left|\frac{i}{\eta} e^{-2 i \sigma_{0}}\left\{\sin \left(\frac{\theta}{2}\right)\right\}^{2(i \eta+1)} \sum_{0}^{\infty}(2 \ell+1) e^{2 i \sigma_{\ell}}\left(S_{\ell}-1\right) P_{\ell}(\cos \theta)\right|^{2} .
$$

Here, the Sommerfeld parameter $\eta=\frac{\mu}{\hbar^{2}} \frac{Z_{1} Z_{2} e^{2}}{k}$ defined in terms of the wave number $\mathrm{k}$, reduced mass $\mu$, and proton numbers $\mathrm{Z}_{1}$ and $\mathrm{Z}_{2}$ of the two interacting nuclei with the 
charge value $\mathrm{e}^{2}=1.4398 \mathrm{MeV}$ fm. Further, $\sigma_{0}$ stands for s-wave Coulomb phase-shift and is expressed as

$$
\sigma_{0}=\frac{\pi}{4}+\eta \log \eta-\eta-\frac{1}{12 \eta}-\frac{1}{360 \eta^{3}}-\frac{1}{1260 \eta^{5}} .
$$

The Coulomb phase-shift, $\sigma_{\ell}$, for higher partial waves is evaluated using

$$
\sigma_{\ell}=\sigma_{\ell-1}+\tan ^{-1} \frac{\eta}{\ell}
$$

$\mathrm{P}_{\ell}(\cos \theta)$ stands for the Legendre polynomials. For the total reaction cross section one can use the formula

$$
\sigma_{R}=\frac{\pi}{k^{2}} \sum_{\ell}(2 \ell+1)\left(1-\left|S_{\ell}\right|^{2}\right)
$$

In the optical model potential $\mathrm{V}(\mathrm{r})$ given by (2) for the collision of two nuclei of mass numbers $\mathrm{A}_{1}$ and $\mathrm{A}_{2}$ and proton number $\mathrm{Z}_{1}$ and $\mathrm{Z}_{2}$, the complex nuclear potential $\mathrm{V}_{N}(\mathrm{r})=\mathrm{V}_{N}^{R}(\mathrm{r})+\mathrm{iV}_{N}^{I}(\mathrm{r})$, the sum of real part $\mathrm{V}_{N}^{R}(r)$ and imaginary part $\mathrm{V}_{N}^{I}(r)$.

We express

$$
\begin{aligned}
V_{N}^{R}(r) & =-V_{0} \frac{1+\delta \exp \left[-\left(r / R_{v}\right)^{2}\right]}{\left\{1+\exp \left[\left(r-R_{s}\right) / 2 a_{s}\right]\right\}^{2}} \\
V_{N}^{I}(r) & =-\frac{W_{0}}{\left\{1+\exp \left[\left(r-R_{I}\right) / 2 a_{I}\right]\right\}^{2}}
\end{aligned}
$$

The radii $\mathrm{R}_{v}, \mathrm{R}_{s}$ and $\mathrm{R}_{I}$ are expressed as $\mathrm{R}_{v}=r_{v}\left(A_{1}^{1 / 3}+A_{2}^{1 / 3}\right), \mathrm{R}_{s}=r_{s}\left(A_{1}^{1 / 3}+A_{2}^{1 / 3}\right)$, and $\mathrm{R}_{I}=r_{I}\left(A_{1}^{1 / 3}+A_{2}^{1 / 3}\right)$, respectively in terms of distance parameters $\mathrm{r}_{v}, \mathrm{r}_{s}$, and $\mathrm{r}_{I}$ in fermi units.

The parameters $\mathrm{a}_{s}$ and $a_{I}$ stand for the slope of the potentials for the real and the imaginary parts, respectively. The depth parameters $\mathrm{V}_{0}>0$ and $\mathrm{W}_{0}>0$ and they are in energy $(\mathrm{MeV})$ units. In the real part $\mathrm{V}_{N}^{R}(r)(10)$, there is a parameter $\delta$ which decides the depth as $\mathrm{V}_{0}(1+\delta)$ near the origin. The use of these potentials in squared Woods-Saxon form has been found successful in the description of $\alpha+{ }^{16} O$ elastic scattering and $\alpha$-cluster structure in ${ }^{20} \mathrm{Ne}$ by Michel et al., [20]. Thus, the real nuclear potential, $\mathrm{V}_{N}^{R}(r)(10)$ is a five-parameter co-ordinate dependent expression with the adjustable parameters $\mathrm{V}_{0}, \mathrm{r}_{v}, \mathrm{r}_{s}$, $\mathrm{a}_{s}$, and $\delta$. The imaginary nuclear potential, $\mathrm{V}_{N}^{I}(r)(11)$ is a three-parameter formula with the parameters $\mathrm{W}_{0}, \mathrm{r}_{I}$, and $\mathrm{a}_{I}$ which are also adjustable.

The Coulomb potential, $\mathrm{V}_{C}(r)$, based on homogeneous charge distributions is expressed as

$$
V_{C}(r)=\left\{\begin{array}{cl}
\frac{Z_{1} Z_{2} e^{2}}{2 R_{C}}\left(3-\frac{r^{2}}{R_{C}^{2}}\right), & \text { if } \mathrm{r} \leq \mathrm{R}_{\mathrm{C}} \\
\frac{Z_{1} Z_{2} e^{2}}{r}, & \text { if } \mathrm{r}>\mathrm{R}_{\mathrm{C}}
\end{array}\right.
$$


where radius parameter $\mathrm{R}_{C}=\mathrm{r}_{C}\left(A_{1}^{1 / 3}+A_{2}^{1 / 3}\right)$ with $\mathrm{r}_{C} \simeq 1.2 \mathrm{fm}$.

Thus, the complete OMP, $\mathrm{V}(\mathrm{r})(2)$, is specified by altogether nine parameters $\mathrm{V}_{0}, \mathrm{r}_{s}, \mathrm{a}_{s}$, $\delta, \mathrm{r}_{v}, \mathrm{r}_{C}, \mathrm{~W}_{0}, \mathrm{r}_{I}$, and $\mathrm{a}_{I}$.

\section{A. Poles of S-matrix for resonance and decay rate}

It may be mentioned here that the real nuclear potential $\mathrm{V}_{N}^{R}(r)$ given by (10) in combination with the Coulomb potential $\mathrm{V}_{C}(r)(12)$ and the centrifugal term $\mathrm{V}_{\ell}(r)=\frac{\hbar^{2}}{2 \mu} \frac{\ell(\ell+1)}{r^{2}}$, generates a repulsive barrier in the outer region with a prominent pocket in the inner side in each partial wave trajectory specified by $\ell=0,1,2,3, \ldots \ldots$. The barrier along with the pocket found in a given $\ell$ gradually vanishes with the increase of $\ell$. Such potentials with well defined pockets can generate significant resonances in the reaction. These potential resonances are identified by the poles of the S-matrix as defined below. Representing the S-matrix $\mathrm{S}_{\ell}(3)$ by $\mathrm{S}_{\ell}(\mathrm{k})=\frac{F(k)}{F(-k)}$, as a function of the wave number $\mathrm{k}$, a zero at $\mathrm{k}=\mathrm{k}_{r}-\mathrm{ik}_{i}$ of $F(-k)$ in the lower half of the complex k-plane gives rise to a pole in the $\mathrm{S}_{\ell}(\mathrm{k})$. When $\mathrm{k}_{i}<<\mathrm{k}_{r}$, this corresponds to a resonance state with positive resonance energy $\mathrm{E}_{r}$ and width $\Gamma_{r}:$

$$
\begin{gathered}
E_{r}=\left(\frac{\hbar^{2}}{2 \mu}\right)\left(k_{r}^{2}-k_{i}^{2}\right), \\
\Gamma_{r}=\left(\frac{\hbar^{2}}{2 \mu}\right)\left(4 k_{r} k_{i}\right) .
\end{gathered}
$$

Thus, in the complex energy $\mathrm{E}$ plane $\mathrm{S}_{\ell}(\mathrm{k})$ has a pole at $\mathrm{E}=\mathrm{E}_{r}-i \Gamma_{r} / 2$. The width $\Gamma_{r}$, expressed in energy unit, is related to decay constant $\lambda_{d}$, mean life $\mathrm{T}$ and half-life $\mathrm{T}_{1 / 2}$ through the relation

$$
\frac{1}{\lambda_{d}}=T=T_{1 / 2} / 0.693=\frac{\hbar}{\Gamma_{r}}
$$

\section{B. Decay rate with exact explicit analytic solution}

Let us study the nature of the potential adopted above for the successful explanation of scattering, reaction and decay of an $\alpha+$ nucleus system. In Fig. 1, we plot the real nuclear 
part $V_{N}^{R}(r)$ (10) combined with Coulomb potential $V_{C}(r)$ (12) for $\ell=0$ trajectory with regard to the $\alpha+$ daughter nucleus, $\alpha+{ }^{208} \mathrm{~Pb}$, pair and show it by the dashed curve. It is clearly seen that there is a well defined pocket followed by a prominent barrier with height $\mathrm{V}_{B}=20.27 \mathrm{MeV}$ positioned at $\mathrm{r}=\mathrm{R}_{B}=10.75 \mathrm{fm}$. This potential (dashed curve) is found close to the dotted curve which represents the potential calculated using energy density profiles of nucleons (proton and neutron) in RMF theory [18] except near the origin where the depth of the RMF potential is small. As mentioned in Sec. II, this combined Coulomb-nuclear potential is responsible for generating resonances or quasi-molecular state that eventually decays. In order to match with the above effective potential, we designate the following form

$$
V_{e f f}(r)=H_{0}\left\{\xi_{1}-\left(\xi_{1}-\xi_{2}\right) \rho(r)\right\}
$$

where

$$
\rho(r)=\left[\cosh ^{2}\left(\frac{R_{0}-r}{d}\right)\right]^{-1}
$$

is well known Eckart form factor and the strength parameter $H_{0}>0$. This potential (16) can be solved in the Schrödinger equation exactly. It has five parameters namely $H_{0}, \xi_{1}, \xi_{2}$, $R_{0}$, and $d$. With the values of the radial position of the barrier obtained by using global formula [21]

$$
\mathrm{R}_{B}=r_{0}\left(A_{1}^{1 / 3}+A_{2}^{1 / 3}\right)+2.72 \mathrm{fm}
$$

with $r_{0}=1.07 \mathrm{fm}$, the height of the barrier

$$
\mathrm{V}_{B}=\frac{Z_{1} Z_{2} e^{2}}{R_{B}}\left(1-\frac{a_{g}}{R_{B}}\right)
$$

with $a_{g}=0.63 \mathrm{fm}$ and setting $\mathrm{R}_{0}=\mathrm{R}_{B}, \mathrm{H}_{0} \xi_{2}=\mathrm{V}_{B}$, depth $H_{0} \xi_{1}=-100 \mathrm{MeV}$, and diffuseness $\mathrm{d}=9.63 \mathrm{fm}$, the effective potential $\mathrm{V}_{\text {eff }}(r)(16)$ is shown by a solid curve in Fig. 1 in the spatial region $0<r<R_{B}$. As we see, it closely matches, in region $0<r<R_{B}$, with the dashed curve that represents the Coulomb+nuclear optical potential for $\ell=0$ described above.

For the potential expressed by Eq. (16), the s-wave radial Schrödinger equation can be written as

$$
\frac{d^{2} u}{d r^{2}}+\left[\kappa^{2}-i k_{0}^{2}(1+i \xi) \rho(r)\right] u=0
$$

where

$$
\begin{aligned}
& \kappa^{2}=k^{2}-k_{0}^{2} \xi_{1}, \\
& \xi=\xi_{1}-\xi_{2},
\end{aligned}
$$


$k_{0}^{2}=\frac{2 \mu}{\hbar^{2}} H_{0}$

The exact solution of the above Eq. (17) is given as

$$
u(r)=A Z^{\frac{1}{2} \kappa d} F(a, b, c, Z)+B Z^{-\frac{1}{2} \kappa d} F\left(a^{\prime}, b^{\prime}, c^{\prime}, Z\right)
$$

where $\mathrm{Z}=\left[\cosh ^{2} \frac{R_{0}-r}{d}\right]^{-1}$ and $\mathrm{F}(\mathrm{a}, \mathrm{b}, \mathrm{c}, \mathrm{Z})$ is the hyper-geometric function. The other terms are

$$
\begin{gathered}
a=\frac{1}{2}(\lambda+i \kappa d), \quad b=\frac{1}{2}(1-\lambda+i \kappa d), \quad c=1+i \kappa d \\
a^{\prime}=\frac{1}{2}(\lambda-i \kappa d), \quad b^{\prime}=\frac{1}{2}(1-\lambda-i \kappa d), \quad c^{\prime}=1-i \kappa d \\
\lambda=\frac{1}{2}-\frac{1}{2}\left[1-i\left(2 k_{0} d\right)^{2}(i \xi)\right]^{1 / 2}
\end{gathered}
$$

Using the boundary condition

$$
u(r=0)=0
$$

we get

$$
Z(r=0)=Z_{0}=\frac{1}{\cosh ^{2} \frac{R_{0}}{d}}
$$

and

$$
C=-\frac{B}{A}=Z_{0}^{i \kappa d} \frac{F\left(a, b, c, Z_{0}\right)}{F\left(a^{\prime}, b^{\prime}, c^{\prime}, Z_{0}\right)} .
$$

For $\cosh ^{2} \frac{R_{0}}{d}>>1, Z_{0}<<1$,

$$
C \simeq \exp \left(-2 i \kappa\left[R_{0}-d \ln 2\right]\right)
$$

The logarithmic derivative of the wave function at $\mathrm{r}=R_{0}$ is given by

$$
f\left(R_{0}\right)=\left.\frac{d u / d r}{u}\right|_{r=R_{0}}=\frac{2}{d} \frac{\frac{\Gamma(c)}{\Gamma(a) \Gamma(b)}-C \frac{\Gamma\left(c^{\prime}\right)}{\Gamma\left(a^{\prime}\right) \Gamma\left(b^{\prime}\right)}}{\frac{\Gamma(c)}{\Gamma(c-a) \Gamma(c-b)}-C \frac{\Gamma\left(c^{\prime}\right)}{\Gamma\left(c^{\prime}-a^{\prime}\right) \Gamma\left(c^{\prime}-b^{\prime}\right)}} .
$$

In the region $r>R_{0}=R_{B}$ where the potential is pure Coulombic, the Coulomb wave functions (regular $F_{0}$ and irregular $G_{0}$ ) and their derivatives $\left(F_{0}^{\prime}\right.$ and $\left.G_{0}^{\prime}\right)$ for $\ell=0$ case are expressed as [22]

$$
\begin{gathered}
F_{0}=\frac{1}{2} \beta \exp (\alpha), \quad F_{0}^{\prime}=\left(\beta^{-2}+\frac{1}{8 \eta} t^{-2} \beta^{4}\right) F_{0}, \\
G_{0}=\frac{\beta}{\exp (\alpha)}, \quad G_{0}^{\prime}=\left[-\beta^{-2}+\frac{1}{8 \eta} t^{-2} \beta^{4}\right] G_{0}, \\
t=\frac{\rho}{2 \eta}, \quad \beta=\left[\frac{t}{1-t}\right]^{\frac{1}{4}}, \\
\alpha=2 \eta\left([t(1-t)]^{\frac{1}{2}}+\arcsin t^{\frac{1}{2}}-\frac{1}{2} \pi\right),
\end{gathered}
$$




$$
k=\sqrt{\frac{2 \mu}{\hbar^{2}} E}, \quad \rho=k R_{0}, \quad \eta=\frac{\mu}{\hbar^{2}} \frac{Z_{1} Z_{2} e^{2}}{k} .
$$

By requirement of continuity at $\mathrm{r}=R_{0}$, the wave functions and their derivatives are matched at $\mathrm{r}=R_{0}$ to obtain the scattering matrix denoted by $\mathrm{S}(\mathrm{k})$ as

$$
S(k)=\frac{2 i k F_{0}^{\prime}-2 i F_{0} f\left(R_{0}\right)+f\left(R_{0}\right)\left[G_{0}+i F_{0}\right]-k\left[G_{0}^{\prime}+i F_{0}^{\prime}\right]}{f\left(R_{0}\right)\left[G_{0}+i F_{0}\right]-k\left[G_{0}^{\prime}+i F_{0}^{\prime}\right]},
$$

where $f\left(R_{0}\right)$ is given by $(25)$. A pole of $\mathrm{S}(\mathrm{k})$ arising from the zero of the denominator of $\mathrm{S}(\mathrm{k})$ (31) in the lower half of complex k-plane gives us the resonance energy equal to Q-value and the decay half-life as described in Sec. II.

\section{RESULTS AND DISCUSSION}

In the application of the above formulation to the explanation of measured data with regard to events namely scattering, reaction and $\alpha$-decay in a $\alpha+$ daughter nucleus system, we select the $\alpha+{ }^{208} \mathrm{~Pb}$ reaction which has been subjected to extensive experiments for the measurements of elastic scattering cross section, reaction cross section, and rate of decay of $\alpha$ particle from the parent ${ }^{212}$ Po nucleus.

The values of the nine potential parameters we use for the total optical model potential for this reaction are $\mathrm{V}_{0}=22 \mathrm{MeV}, \mathrm{r}_{s}=1.27 \mathrm{fm}, \mathrm{a}_{s}=0.62 \mathrm{fm}, \mathrm{r}_{v}=0.66 \mathrm{fm}, \delta=3.5, \mathrm{r}_{C}=1.2 \mathrm{fm}$, $\mathrm{W}_{0}=5 \mathrm{MeV}, \mathrm{a}_{I}=0.28 \mathrm{fm}$, and $\mathrm{r}_{I}=1.2 \mathrm{fm}$ for $\mathrm{E}_{l a b}=19 \mathrm{MeV}, \mathrm{r}_{I}=1.3 \mathrm{fm}$ for $\mathrm{E}_{l a b}=20 \mathrm{MeV}$, $\mathrm{r}_{I}=1.37 \mathrm{fm}$ for $\mathrm{E}_{l a b}=22 \mathrm{MeV}$ where $\mathrm{E}_{l a b}$ stands for incident energy in the laboratory.

Using the S-matrix $S_{\ell}(3)$ in the expression (6), we obtain the results of angular variation of differential elastic scattering cross section $\sigma_{S c}$ in ratio to Rutherford scattering cross section $\sigma_{R u}$ and compare them (full curves) with the corresponding measured data (solid circles) obtained from Ref. [23] in Fig. 2. It is found that the fitting of the data at three different energies around the s-wave barrier height $(=20.27 \mathrm{MeV})$ is quite good. With this, the test for the authenticity of the nuclear optical potential adopted in the present analysis is successful. Now the same potential is to be tested for the explanation of reaction cross section and also the result of $\alpha$-decay rate.

By using the expression (9), the total reaction cross section $\sigma_{R}$ as a function of bombarding energy are obtained and they are shown by a solid curve in Fig. 3 and compared with 
the corresponding experimental data represented by solid circles [23] in this Fig. 3. It is clearly seen that the explanation of the data by our calculated results (solid curve) is quite satisfactory at different energies around the barrier. It may be pointed out here that the results of $\sigma_{R}$ at low $(<20 \mathrm{MeV})$ incident energies analyzed here are sometimes considered as fusion cross sections [15].

Coming to the calculation of resonance energy and the decay width through the poles of S-matrix, we first find out the resonance energy equal to the Q-value of $\alpha$-decay from the position of a peak in the variation of the result of $\sigma_{R}$ as a function of energy by using the same set of potential parameters which is found successful in explaining the elastic and $\sigma_{R}$ data mentioned above. In order to obtain the resonance energy exactly equal to the Q-value we may need to marginally vary the depth or the diffuseness parameter of the real nuclear potential which eventually does not affect the results of elastic or reaction cross sections obtained earlier. This resonance energy equal to the Q-value is used as a trial value of the real part of the pole position $\left(k_{r}^{0}\right)$ of the S-matrix, $S_{\ell}(3)$. The trial value of the imaginary part $\left(k_{i}^{0}\right)$ of the pole position is taken to be a very small value $(\approx 0.001 \mathrm{MeV})$ to begin with. Starting with these trial values, Newton-Raphson iterative technique is used to obtain the zero of the Jost function of the Coulomb-nuclear S-matrix, $S_{\ell}(3)$, which corresponds to the resonance or quasi-bound state pole. From this pole, using Eq. (13), the resonance energy $\mathrm{E}_{r}$ is obtained to represent the Q-value. The corresponding result of width is obtained by using (14) and from this, using relation (15), the value of decay half-life denoted by $T_{1 / 2}^{(O M P)}$ is obtained within the framework of optical model calculation. In the case of $\alpha$-emitter ${ }^{212} \mathrm{Po}$ with $\mathrm{Q}$-value $Q_{\alpha}=8.954 \mathrm{MeV}$, we find $T_{1 / 2}^{(O M P)}=2.89 \times 10^{-7} \mathrm{~s}$ which is very close to the experimental value of half-life $T_{1 / 2}^{(\text {expt })}=2.99 \times 10^{-7} s$. For other $\alpha+$ daughter nucleus pairs, we can use the same optical model potential parameters used above in the analysis of the $\alpha+{ }^{208} \mathrm{~Pb}$ pair and estimate the $\alpha$-decay half-life $T_{1 / 2}^{(O M P)}$ of the parent nuclei. Having fixed all other parameters namely $\mathrm{V}_{0}=22 \mathrm{MeV}, \mathrm{r}_{s}=1.27 \mathrm{fm}, \mathrm{a}_{s}=0.62 \mathrm{fm}$, and $\mathrm{r}_{C}=1.2 \mathrm{fm}$, one has to marginally vary the value of the parameter $\mathrm{r}_{v}$ around $0.66 \mathrm{fm}$ and that of $\delta$ around 3 to generate the resonance energy exactly at the Q-value of a given pair. This formulation can easily be applied to estimate the results of decay half-lives of $\alpha$ particle emitted out with some angular momentum $\ell>0$. For this, one has to simply generate the resonance at the energy equal to the given Q-value of decay in the specified partial wave trajectory $\ell$ by the variation of $\mathrm{r}_{v}$ and $\delta$ outlined above. We calculate the results of decay half-life in decimal 
logarithm, $\left(\log _{10} T_{1 / 2}^{(O M P)}\right)$, for several $\alpha$-emitters in the list of Polonium (Po) isotopes for $\ell=0$ state and compare them with the corresponding experimental data denoted by $\log _{10} T_{1 / 2}^{(e x p t)}$ in Table I. We find that our calculated results are close to the respective measured data in most cases of $\alpha+$ daughter nucleus pairs.

We now calculate $T_{1 / 2}$ of the $\alpha+$ daughter system from the resonance pole of the S-matrix, $\mathrm{S}(\mathrm{k})$ (31) derived by using exact wave functions of Coulomb-nuclear interaction. The same procedure adopted above in the OMP calculation is used here to locate the pole of $\mathrm{S}(\mathrm{k})$ depicting the resonance at the energy equal to the Q-value of decay. In this case, the diffuseness parameter 'd' of the potential (16) is varied to obtain the above situation of resonance at the $\mathrm{Q}$-value. From this pole of $\mathrm{S}(\mathrm{k})$ we derive decay $T_{1 / 2}$ by using formula (15) and denote the results by $T_{1 / 2}^{(a n a l)}$ as it is based on exact analytical solutions unlike those used in the derivation of $T_{1 / 2}^{(O M P)}$ from the pole of $\mathrm{S}_{\ell}(3)$ obtained within the OMP potential calculation. In this potential model calculation, we obtain the result of $T_{1 / 2}^{(\text {anal })}=$ $3.02 \times 10^{-7} s$ for the $\alpha$-decay of the ${ }^{212} \mathrm{Po}$ nucleus which is very close to the experimental result $T_{1 / 2}^{(e x p t)}=2.99 \times 10^{-7} s$ with $Q_{\alpha}=8.954 \mathrm{MeV}$. Also, it is found that the result of $T_{1 / 2}^{(\text {anal })}$ is very close to the value of $T_{1 / 2}^{(O M P)}=2.89 \times 10^{-7} \mathrm{~S}$. This closeness between our calculated results of $T_{1 / 2}^{(\text {anal })}$ and $T_{1 / 2}^{(O M P)}$ indicates that the effective potential (16) with parameters $\mathrm{r}_{0}=1.07 \mathrm{fm}, \mathrm{a}_{g}=0.63 \mathrm{fm}$ and $\mathrm{d}=9.63 \mathrm{fm}$ is a good approximation for the Coulomb-nuclear interaction potential of the $\alpha+{ }^{208} \mathrm{~Pb}$ pair for the estimate of decay half-life using exact solution of the potential for the S-matrix and its resonance pole. As the potential (16) uses global formula for its parameters for barrier position and height, one can use this in the cases of other $\alpha+$ daughter nucleus pairs with some variation of the diffuseness parameter $\mathrm{d}$ around $9 \mathrm{fm}$ to generate the resonance at the energy equal to the Q-value of the of the given pair and obtain the result of decay half-life from the pole of this resonance. We calculate the results $\left(T_{1 / 2}^{(\text {anal })}\right)$ for several $\alpha$-emitters in the list of Polonium (Po) isotopes for $\ell=0$ state and compare them with the corresponding experimental data in Table I. We find that our calculated results of half-life in decimal logarithm, $\left(\log _{10} T_{1 / 2}^{(a n a l)}\right)$, having closely reproduced the results $\left(\log _{10} T_{1 / 2}^{(O M P)}\right)$ obtained in OMP calculation above are found close to the respective measured data $\log _{10} T_{1 / 2}^{(e x p t)}$ in most cases of $\alpha$-emitters.

From the above analysis we learn that for the emission of $\alpha$ particle in $\ell=0$ situation, instead of using poles from $\mathrm{S}_{\ell}$ (3) which requires tedious numerical calculations of wave functions of the full OMP, it is okay to use the simple poles of $\mathrm{S}(\mathrm{k})$ (31) which is expressed 
analytically in terms of exact solution of a global potential and analytical Coulomb wave functions, and estimate the results of decay half-life, $T_{1 / 2}^{(a n a l)}$, for the explanation of experimental decay rate in various $\alpha+$ daughter nucleus pairs. In Table II, we present the results of $T_{1 / 2}^{(\text {anal })}$ for several $\alpha$-emitters. On comparison with the corresponding experimental data denoted by $T_{1 / 2}^{(\text {expt })}$ in the same Table II, we find that our results provide satisfactory explanation of the measured ones in most cases of the $\alpha$-emitters.

For emission of $\alpha$ particle with $\ell>0$, we have to use the full optical potential in different trajectories specified by $\ell$ s and estimate the results, $T_{1 / 2}^{(O M P)}$, from the poles of $\mathrm{S}_{\ell}(3)$ described above. In Table III, we present our results of half-life, $\log _{10} T_{1 / 2}^{(O M P)}$, in decimal logarithm for several $\alpha$-emitters in different $\ell>0$ situations and compare them with the corresponding experimental results denoted by $\log _{10} T_{1 / 2}^{(e x p t)}$. We find that the explanation of the measured data by our calculated ones is quite satisfactory in most cases of decay. In few cases, namely ${ }_{73}^{159} X,{ }_{91}^{214} X,{ }_{91}^{229} X$, and ${ }_{101}^{257} X$, the angular momenta assigned in the experimental results are different from the $\ell$ s we need to consider for proper fitting of the $Q_{\alpha}$-values and the corresponding half-lives. These $\ell$ s decided by our systematic calculation are noted within brackets () by the side of the measured $\ell$ s for the above four nuclei.

Having obtained successes in the explanation of all the three $\alpha$ induced nuclear collision events: elastic scattering, reaction and decay by the use of the nuclear potential (10) in squared Woods-Saxon form, the following few words are in order in favour of this potential. (i) The potential has a surface part defined by the parameters $V_{0}, r_{s}$, and $a_{s}$ as in normal Woods-Saxon form and it takes care of proper description of the measured data of elastic scattering which is a surface phenomenon.

(ii) There is a volume part in this potential expression (10) governed by the parameters $\mathrm{r}_{v}$ and $\delta$ which controls the diffuseness of the potential in the interior side. Interestingly, variation of these parameters does not disturb much the fitting of the elastic scattering cross section provided by the surface part stated in point (i). On the other hand, by selecting some values $\mathrm{r}_{v} \sim 0.66 \mathrm{fm}$ and $\delta \sim 3$, we, in combination with the repulsive Coulomb part, find an effective potential which is slowly falling in nature towards the left hand side of the Coulomb barrier. This bulging character of the Coulomb+nuclear potential provides all the remarkable explanation of the experimental data of $\alpha$-decay half-lives and also the reaction cross sections at energies near and below the barrier in large number of $\alpha+$ daughter nucleus collision events. From the successful application of this form of nuclear potential 
we understand that the volume part deciding the diffuseness of the Coulomb barrier in the interior side is the life-line for the explanation of the decay rate and reaction or fusion cross section in the collision of $\alpha+$ nucleus system.

(iii) This short of potential with less diffuseness in the interior side of the Coulomb barrier potential is consistent with the potential calculated using density profiles of nucleons in the RMF theory [18].

\section{SUMMARY AND CONCLUSION}

Considering the process of decay of an $\alpha$ particle from a parent nucleus as a two-body quantum collision of $\alpha+$ daughter nucleus pair, three events namely decay, elastic scattering and reaction (fusion) are addressed in one platform within the framework of threedimensional optical model potential scattering (S-matrix) theory. A novel expression for the nuclear potential in squared Woods-Saxon form is adopted for the nucleus-nucleus collision. Using the S-matrix of the complex nuclear plus electrostatic potentials, the measured data of elastic scattering and reaction cross sections are explained to proof the genuineness of the potential. From the poles of the same S-matrix in the complex momentum plane, we extract the energy and width of the resonance state akin to the decaying state of emission of $\alpha$ particle and from this width the result of decay half-life of the $\alpha$-emission is obtained to account for the experimental data of half-life in the cases of large number of $\alpha$-emitters including heavy and super-heavy nuclei.

In this comprehensive analysis of three physical phenomena, we find that the versatile form of nuclear potential adopted by us in this paper, by virtue of its surface part, explains data of elastic scattering cross section and by the help of its volume part controlling the diffuseness of the potential in the interior side, decides the results of reaction cross section and decay rate yielding good explanation of respective measured data.

The sum of the above nuclear potential (real part) with the Coulomb potential based on homogeneous distribution of charges for s-wave is closely represented by an analytical expression as a function of radial distance $r$ which is solved exactly to express the S-matrix in terms of the explicit analytical Schrödinger solutions and Coulomb wave functions. From the resonant poles of this well defined S-matrix of a global soluble potential, the results 
of decay half-lives are obtained to explain the corresponding experimental data in several heavy as well as super-heavy $\alpha$ emitting nuclei giving rise to satisfactory explanation of the data.

In conclusion, we believe that the emission of $\alpha$ particle from a radioactive nucleus is governed by the fundamental principle of quantal decay of charged particle from a resonance state generated by a two-body ( $\alpha+$ daughter nucleus) potential that describes the elastic and reaction cross sections of the $\alpha+$ daughter nucleus collision. And the width of the resonant pole of the S-matrix of the potential yields the result of decay half-life.

Further, the S-matrix of the full optical model calculation involving tedious numerical computation for wave functions can be replaced by a S-matrix which is expressed in terms of exact analytical solutions of a soluble potential that closely represents the real part of the potential describing elastic scattering data and expressions of Coulomb wave functions, and the resonant poles of this S-matrix in the complex momentum plane can be used to give satisfactory results of $\alpha$-decay half-lives.

\section{ACKNOWLEDGMENTS}

We would like to thank Bharat Kumar, Ph.D. Scholar, Institute of Physics, Bhubaneswar,

India, for supplying the results of the potential for the $\alpha+{ }^{208} \mathrm{~Pb}$ system using RMF theory. We acknowledge the research facilities extended to us by the Institute of Physics, Bhubaneswar, India. 
[1] Peter Mohr, Phys. Rev. C 73, 031301 (R) (2006).

[2] C. Xu and Z. Ren, Nucl. Phys. A 753, 174 (2005).

[3] V. Yu. Denisov and H. Ikezoe, Phys. Rev. C 72, 064613 (2005).

[4] Y. K. Gambhir, A. Bhagwat, and M. Gupta, Phys. Rev. C 71, 037301 (2005).

[5] Z. A. Dupre' and J. J. Bürvenich, Nucl. Phys. A 767, 81 (2006).

[6] T. Dong and Z. Ren, Eur. Phys. J. A 26, 69 (2005).

[7] T. Dong and Z. Ren, Phys. Rev. C 72, 064331 (2005).

[8] P. R. Chowdhury, C. Samanta, and D. N. Basu, Phys. Rev. C 73, 014612 (2006).

[9] B. Sahu, Phys. Rev. C 78, 044608 (2008); Phys. Rev. C 84, 037607 (2011); Phys. Rev. C 85, 057601 (2012).

[10] B. Sahu, Y. K. Gambhir, and C. S. Shastry, Mod. Phys. Lett. A 25, 535 (2010).

[11] B. Sahu and Swagatika Bhoi, Phys. Rev. C 93, 044301 (2016).

[12] B. Sahu, R. Paira, and B. Rath, Nucl. Phys. A 908, 40 (2013).

[13] S. A. Gurvitz and G. kälbermann, Phys. Rev. Lett. 59, 262 (1987).

[14] Y. K. Gambhir, A. Bhagwat, M. Gupta, and A. K. Jain, Phys. Rev. C 68, 044316 (2003).

[15] A. Bhagwat and Y. K. Gambhir, J. Phys. G: Nucl. Part. Phys. 35, 065109 (2008).

[16] B. Sahu, G. S. Mallick, B. B. Sahu, S. K. Agarwalla, and C. S. Shastry, Phys. Rev. C 77, 024604 (2008).

[17] B. Sahu and B. Sahu, Int. J. Mod. Phys. E 21, 1250067 (2012).

[18] B. Kumar, S. K. Biswal, S. K. Singh, C. Lahiri, and S. K. Patra, Int. J. Mod. Phys. E 25, 1650020 (2016).

[19] B. Sahu, B. B. Sahu, and S. K. Agarwalla, Pramana 70, 27 (2008).

[20] F. Michel, J. Albinski, P. Belery, Th. Delbar, Gh. Gregoire, B. Tasiaux, and G. Reidemeister, Phys. Rev. C 28, 1904 (1983).

[21] R. A. Broglia, A. Winther, Heavy-Ion Reactions Lecture Notes (Addison-Wesley, Redwood, 1981).

[22] M. Abramowitz and I. A. Stegun, Handbook of Mathematical Functions (Dover, New York, 1965), p. 542.

[23] A. R. Barnett and J. S. Lilley, Phys. Rev. C 9, 2010 (1974). 
[24] Yuejiao Ren and Zhongzhou Ren, Phys. Rev. C 85, 044608 (2012).

[25] Dongdong Ni and Zhongzhou Ren, Nucl. Phys. A 825, 145 (2009).

[26] G. Royer, Nucl. Phys. A 848, 279 (2010). 
TABLE I. Comparison of experimental results of $\alpha$-decay half-life in decimal logarithm, $\log _{10} T_{1 / 2}^{(\text {expt })}$ (third column) with the calculated results $\log _{10} T_{1 / 2}^{(O M P)}$ (fourth column) obtained from the poles of S-matrix, $\mathrm{S}_{\ell}(3)$, and $\log _{10} T_{1 / 2}^{(\text {anal) }}$ (fifth column) from the poles of analytical S-matrix $\mathrm{S}(\mathrm{k})$ given by (31). In the sixth column, the values of diffuseness parameter 'd' used in the calculation of $\log _{10} T_{1 / 2}^{(\text {anal })}$ are listed. For the calculation of $\log _{10} T_{1 / 2}^{(O M P)}$, the values of real optical potential parameters $\mathrm{V}_{0}=22 \mathrm{MeV}, \mathrm{r}_{s}=1.27 \mathrm{fm}, \mathrm{a}_{s}=0.62 \mathrm{fm}$, and $\mathrm{r}_{C}=1.2 \mathrm{fm}$ are kept same

for all nuclei, and the values of the parameters $\mathrm{r}_{v}$ and $\delta$ are varied around $0.66 \mathrm{fm}$ and 3 , respectively, to exactly reproduce the experimental $Q_{\alpha}$ value of a given alpha decaying isotope.

Experimental data are obtained from Ref. [24].

\begin{tabular}{cccccc}
\hline \hline Decay & $Q_{\alpha}^{(\text {expt })}$ & $\log _{10} \mathrm{~T}_{1 / 2}^{(\text {expt })}$ & $\log _{10} \mathrm{~T}_{1 / 2}^{(\text {OMP })}$ & $\log _{10} \mathrm{~T}_{1 / 2}^{(\text {anal })}$ & $\mathrm{d}$ \\
& $(\mathrm{MeV})$ & $(\mathrm{s})$ & $(\mathrm{s})$ & $(\mathrm{s})$ & $(\mathrm{fm})$ \\
\hline${ }^{218} \mathrm{Po} \rightarrow{ }^{214} \mathrm{~Pb}$ & 6.115 & 2.27 & 2.36 & 2.28 & 7.9070 \\
${ }^{216} \mathrm{Po} \rightarrow{ }^{212} \mathrm{~Pb}$ & 6.906 & -0.84 & -0.75 & -0.89 & 7.9915 \\
${ }^{214} \mathrm{Po} \rightarrow{ }^{210} \mathrm{~Pb}$ & 7.833 & -3.38 & -3.71 & -3.97 & 8.0996 \\
${ }^{212} \mathrm{Po} \rightarrow{ }^{208} \mathrm{~Pb}$ & 8.954 & -6.52 & -6.68 & -6.52 & 9.6357 \\
${ }^{210} \mathrm{Po} \rightarrow{ }^{206} \mathrm{~Pb}$ & 5.407 & 7.08 & 6.83 & 6.40 & 8.9432 \\
${ }^{208} \mathrm{Po} \rightarrow{ }^{204} \mathrm{~Pb}$ & 5.215 & 7.96 & 7.96 & 7.47 & 8.8738 \\
${ }^{206} \mathrm{Po} \rightarrow{ }^{202} \mathrm{~Pb}$ & 5.327 & 7.14 & 6.97 & 6.88 & 8.8554 \\
${ }^{204} \mathrm{Po} \rightarrow{ }^{200} \mathrm{~Pb}$ & 5.485 & 6.28 & 6.14 & 6.05 & 8.8442 \\
${ }^{202} \mathrm{Po} \rightarrow{ }^{198} \mathrm{~Pb}$ & 5.701 & 5.15 & 5.06 & 4.96 & 8.8425 \\
${ }^{200} \mathrm{Po} \rightarrow{ }^{196} \mathrm{~Pb}$ & 5.981 & 3.74 & 3.74 & 3.64 & 8.8511 \\
${ }^{198} \mathrm{Po} \rightarrow{ }^{194} \mathrm{~Pb}$ & 6.309 & 2.27 & 2.30 & 2.19 & 8.8677 \\
${ }^{196} \mathrm{Po} \rightarrow{ }^{192} \mathrm{~Pb}$ & 6.657 & 0.77 & 0.90 & 0.79 & 8.8765 \\
${ }^{194} \mathrm{Po} \rightarrow{ }^{190} \mathrm{~Pb}$ & 6.987 & -0.41 & -0.31 & -0.43 & 8.9045 \\
${ }^{190} \mathrm{Po} \rightarrow{ }^{186} \mathrm{~Pb}$ & 7.693 & -2.61 & -2.65 & -2.77 & 8.9461 \\
\hline \hline
\end{tabular}


TABLE II. Comparison of the experimental $\alpha$-decay half-lives with the calculated ones for nuclei with the neutron number $\mathrm{N}>126$. The first and second columns denote the elemental symbol and the mass number of the parent nucleus. The third and fourth columns are, respectively, the experimental decay energies $\left(Q_{\alpha}\right.$ values) and half-lives $\left(T_{1 / 2}^{(e x p t)}\right)$ of $\alpha$ decay obtained from Ref. [25]. The half-lives, $T_{1 / 2}^{(\text {anal })}$, calculated from the poles of analytical S-matrix $\mathrm{S}(\mathrm{k})$ given by (31) are presented in the fifth column. In the sixth column, the values of diffuseness parameter 'd' used in the calculation are listed.

\begin{tabular}{|c|c|c|c|c|c|}
\hline Elt. & $\mathrm{A}$ & $Q_{\alpha}(\mathrm{MeV})$ & $T_{1 / 2}^{(e x p t)}(\mathrm{s})$ & $T_{1 / 2}^{(\text {anal })}(\mathrm{s})$ & $\mathrm{d}(\mathrm{fm})$ \\
\hline $\mathrm{Pb}$ & 210 & 3.792 & $3.69 \times 10^{16}$ & $3.00 \times 10^{16}$ & 8.73 \\
\hline \multirow[t]{4}{*}{ Po } & 212 & 8.954 & $2.99 \times 10^{-7}$ & $3.02 \times 10^{-7}$ & 9.63 \\
\hline & 214 & 7.833 & $1.64 \times 10^{-4}$ & $1.02 \times 10^{-4}$ & 8.10 \\
\hline & 216 & 6.906 & $1.45 \times 10^{-1}$ & $1.24 \times 10^{-1}$ & 7.99 \\
\hline & 218 & 6.115 & $1.86 \times 10^{2}$ & $1.91 \times 10^{2}$ & 7.90 \\
\hline \multirow[t]{5}{*}{$\mathrm{Rn}$} & 214 & 9.208 & $2.70 \times 10^{-7}$ & $1.22 \times 10^{-7}$ & 8.26 \\
\hline & 216 & 8.200 & $4.50 \times 10^{-5}$ & $5.10 \times 10^{-5}$ & 8.14 \\
\hline & 218 & 7.263 & $3.5 \times 10^{-2}$ & $4.68 \times 10^{-2}$ & 8.03 \\
\hline & 220 & 6.405 & $5.56 \times 10^{1}$ & $9.1 \times 10^{1}$ & 7.93 \\
\hline & 222 & 5.590 & $3.31 \times 10^{5}$ & $6.1 \times 10^{5}$ & 7.85 \\
\hline \multirow[t]{6}{*}{$\mathrm{Ra}$} & 216 & 9.526 & $1.82 \times 10^{-7}$ & $1.05 \times 10^{-7}$ & 8.29 \\
\hline & 218 & 8.546 & $2.56 \times 10^{-5}$ & $3.10 \times 10^{-5}$ & 8.17 \\
\hline & 220 & 7.592 & $1.81 \times 10^{-2}$ & $2.36 \times 10^{-2}$ & 8.06 \\
\hline & 222 & 6.679 & $3.92 \times 10^{1}$ & $5.37 \times 10^{1}$ & 7.96 \\
\hline & 224 & 5.789 & $3.33 \times 10^{5}$ & $5.96 \times 10^{5}$ & 7.86 \\
\hline & 226 & 4.871 & $5.35 \times 10^{10}$ & $12.56 \times 10^{10}$ & 7.76 \\
\hline \multirow[t]{6}{*}{ Th } & 218 & 9.849 & $1.09 \times 10^{-7}$ & $0.89 \times 10^{-7}$ & 8.33 \\
\hline & 220 & 8.953 & $9.70 \times 10^{-6}$ & $1.33 \times 10^{-5}$ & 8.22 \\
\hline & 222 & 8.127 & $2.05 \times 10^{-3}$ & $2.80 \times 10^{-3}$ & 8.13 \\
\hline & 224 & 7.298 & $1.33 \times 10^{0}$ & $1.61 \times 10^{0}$ & 8.03 \\
\hline & 226 & 6.451 & $2.46 \times 10^{3}$ & $3.94 \times 10^{3}$ & 7.94 \\
\hline & 228 & 5.520 & $8.49 \times 10^{7}$ & $1.65 \times 10^{8}$ & 7.84 \\
\hline
\end{tabular}


TABLE II. (continued).

\begin{tabular}{|c|c|c|c|c|c|}
\hline Elt. & $\mathrm{A}$ & $\mathrm{Q}(\mathrm{MeV})$ & $T_{1 / 2}^{(e x p t)}(\mathrm{s})$ & $T_{1 / 2}^{(\text {exat })}(\mathrm{s})$ & $\mathrm{d}(\mathrm{fm})$ \\
\hline \multirow{5}{*}{$\mathrm{U}$} & 230 & 4.770 & $3.12 \times 10^{12}$ & $7.82 \times 10^{12}$ & 7.77 \\
\hline & 232 & 4.082 & $5.69 \times 10^{17}$ & $2.21 \times 10^{18}$ & 7.71 \\
\hline & 222 & 9.500 & $1.40 \times 10^{-6}$ & $2.62 \times 10^{-6}$ & 8.29 \\
\hline & 224 & 8.620 & $9.40 \times 10^{-4}$ & $5.66 \times 10^{-4}$ & 8.18 \\
\hline & 226 & 7.701 & $2.69 \times 10^{-1}$ & $4.14 \times 10^{-1}$ & 8.08 \\
\hline \multirow{13}{*}{$\mathrm{Pu}$} & 228 & 6.803 & $5.75 \times 10^{2}$ & $9.78 \times 10^{2}$ & 7.98 \\
\hline & 230 & 5.993 & $2.67 \times 10^{6}$ & $5.08 \times 10^{6}$ & 7.90 \\
\hline & 232 & 5.414 & $3.20 \times 10^{9}$ & $7.32 \times 10^{9}$ & 7.85 \\
\hline & 234 & 4.858 & $1.09 \times 10^{13}$ & $2.52 \times 10^{13}$ & 7.80 \\
\hline & 236 & 4.673 & $1.00 \times 10^{15}$ & $3.08 \times 10^{15}$ & 7.79 \\
\hline & 238 & 4.270 & $1.78 \times 10^{17}$ & $8.82 \times 10^{17}$ & 7.78 \\
\hline & 232 & 6.716 & $1.71 \times 10^{4}$ & $1.77 \times 10^{4}$ & 7.99 \\
\hline & 234 & 6.310 & $7.73 \times 10^{5}$ & $12.31 \times 10^{5}$ & 7.96 \\
\hline & 236 & 5.867 & $1.30 \times 10^{8}$ & $2.11 \times 10^{8}$ & 7.93 \\
\hline & 238 & 5.593 & $3.90 \times 10^{9}$ & $6.62 \times 10^{9}$ & 7.92 \\
\hline & 240 & 5.256 & $2.84 \times 10^{11}$ & $6.82 \times 10^{11}$ & 7.91 \\
\hline & 242 & 4.985 & $1.52 \times 10^{13}$ & $3.95 \times 10^{13}$ & 7.90 \\
\hline & 244 & 4.666 & $3.17 \times 10^{15}$ & $8.11 \times 10^{15}$ & 7.88 \\
\hline \multirow[t]{5}{*}{$\mathrm{Cm}$} & 240 & 6.398 & $3.30 \times 10^{6}$ & $3.75 \times 10^{6}$ & 8.02 \\
\hline & 242 & 6.216 & $1.90 \times 10^{7}$ & $2.82 \times 10^{7}$ & 8.03 \\
\hline & 244 & 5.902 & $7.48 \times 10^{8}$ & $1.16 \times 10^{9}$ & 8.01 \\
\hline & 246 & 5.475 & $1.82 \times 10^{11}$ & $3.20 \times 10^{11}$ & 7.98 \\
\hline & 248 & 5.162 & $1.43 \times 10^{13}$ & $3.03 \times 10^{13}$ & 7.97 \\
\hline \multirow[t]{5}{*}{$\mathrm{Cf}$} & 240 & 7.719 & $9.09 \times 10^{1}$ & $8.77 \times 10^{1}$ & 8.17 \\
\hline & 242 & 7.517 & $2.62 \times 10^{2}$ & $4.77 \times 10^{2}$ & 8.17 \\
\hline & 244 & 7.329 & $1.55 \times 10^{3}$ & $2.45 \times 10^{3}$ & 8.17 \\
\hline & 246 & 6.862 & $1.62 \times 10^{6}$ & $2.12 \times 10^{5}$ & 8.14 \\
\hline & 248 & 6.361 & $3.54 \times 10^{7}$ & $4.37 \times 10^{7}$ & 8.10 \\
\hline
\end{tabular}


TABLE II. (continued).

\begin{tabular}{cccccc}
\hline \hline Elt. & $\mathrm{A}$ & $Q_{\alpha}(\mathrm{MeV})$ & $T_{1 / 2}^{(\text {expt })}(\mathrm{s})$ & $T_{1 / 2}^{(\text {exat })}(\mathrm{s})$ & $\mathrm{d}(\mathrm{fm})$ \\
\hline \hline \multirow{6}{*}{ Fm } & 250 & 6.128 & $4.88 \times 10^{8}$ & $6.48 \times 10^{8}$ & 8.09 \\
& 252 & 6.217 & $1.02 \times 10^{8}$ & $2.10 \times 10^{8}$ & 8.14 \\
& 254 & 5.927 & $2.04 \times 10^{9}$ & $7.07 \times 10^{9}$ & 8.12 \\
& 246 & 8.374 & $1.55 \times 10^{0}$ & $2.69 \times 10^{0}$ & 8.31 \\
& 250 & 7.557 & $2.28 \times 10^{3}$ & $2.08 \times 10^{3}$ & 8.25 \\
& 252 & 7.153 & $1.09 \times 10^{5}$ & $8.30 \times 10^{4}$ & 8.22 \\
& 254 & 7.308 & $1.37 \times 10^{4}$ & $1.78 \times 10^{4}$ & 8.28 \\
& 256 & 7.027 & $1.35 \times 10^{5}$ & $2.78 \times 10^{5}$ & 8.27 \\
No & 252 & 8.550 & $4.18 \times 10^{0}$ & $3.96 \times 10^{0}$ & 8.38 \\
& 254 & 8.226 & $7.14 \times 10^{1}$ & $4.58 \times 10^{1}$ & 8.37 \\
& 256 & 8.581 & $3.64 \times 10^{0}$ & $2.89 \times 10^{0}$ & 8.45 \\
Rf & 256 & 8.930 & $2.02 \times 10^{0}$ & $1.32 \times 10^{0}$ & 8.46 \\
& 258 & 9.250 & $9.23 \times 10^{-2}$ & $1.36 \times 10^{-1}$ & 8.54 \\
\hline \hline
\end{tabular}


TABLE III. Comparison of experimental values in decimal logarithm $\log _{10} T_{1 / 2}^{(e x p t)}$ of half-life of $\alpha$-decay and corresponding results of present calculation $\log _{10} T_{1 / 2}^{(O M P)}$ obtained from the poles of $\mathrm{S}_{\ell}(3)$ in decimal logarithm. The experimental $Q_{\alpha}$ values, half-lives and $l$ values are obtained from Ref. [26].

\begin{tabular}{|c|c|c|c|c|c|c|c|c|c|}
\hline$\stackrel{A}{Z}$ & $Q_{\alpha}(\mathrm{MeV})$ & $l$ & $\log _{10} T_{1 / 2}^{(e x p t)}(s)$ & $\log _{10} T_{1 / 2}^{(O M P)}(s)$ & $\stackrel{A}{Z}$ & $Q_{\alpha}(\mathrm{MeV})$ & $l$ & $\log _{10} T_{1 / 2}^{(e x p t)}(s)$ & $\log _{10} T_{1 / 2}^{(O M P)}(s)$ \\
\hline $\begin{array}{l}112 \\
53\end{array}$ & 2.990 & 4 & 5.45 & 5.54 & $\begin{array}{l}149 \\
65\end{array}$ & 4.077 & 2 & 4.97 & 4.96 \\
\hline $\begin{array}{l}151 \\
65\end{array}$ & 3.496 & 2 & 8.82 & 8.80 & $\begin{array}{l}159 \\
73\end{array}$ & 5.681 & $5(0)$ & 0.11 & 0.12 \\
\hline $\begin{array}{l}162 \\
73\end{array}$ & 5.010 & 1 & 3.68 & 3.62 & $\begin{array}{l}175 \\
77\end{array}$ & 5.400 & 2 & 3.02 & 3.38 \\
\hline $\begin{array}{l}181 \\
79\end{array}$ & 5.751 & 2 & 3.39 & 3.34 & $\begin{array}{l}191 \\
83\end{array}$ & 6.778 & 5 & 2.85 & 2.96 \\
\hline $\begin{array}{l}193 \\
83\end{array}$ & 6.304 & 5 & 4.50 & 4.22 & $\begin{array}{l}195 \\
83\end{array}$ & 5.832 & 5 & 6.79 & 6.34 \\
\hline $\begin{array}{l}210 \\
85\end{array}$ & 5.631 & 2 & 7.73 & 7.34 & $\begin{array}{l}210 \\
87\end{array}$ & 6.650 & 2 & 2.43 & 2.69 \\
\hline $\begin{array}{l}212 \\
83\end{array}$ & 6.207 & 5 & 4.57 & 4.18 & $\begin{array}{l}212 \\
85\end{array}$ & 7.824 & 5 & -0.42 & -0.42 \\
\hline $\begin{array}{l}212 \\
87\end{array}$ & 6.529 & 2 & 4.10 & 4.10 & ${ }_{83}^{213}$ & 5.982 & 5 & 5.15 & 5.18 \\
\hline $\begin{array}{l}214 \\
83\end{array}$ & 5.621 & 5 & 7.16 & 7.20 & $\begin{array}{l}214 \\
87\end{array}$ & 8.589 & 5 & -2.27 & -2.20 \\
\hline $\begin{array}{l}214 \\
89\end{array}$ & 7.350 & 2 & 1.23 & 1.35 & $\begin{array}{l}214 \\
91\end{array}$ & 8.430 & $4(1)$ & -2.10 & -2.32 \\
\hline $\begin{array}{l}216 \\
89\end{array}$ & 9.235 & 5 & -3.31 & -3.30 & $\begin{array}{l}220 \\
87\end{array}$ & 6.801 & 1 & 1.62 & 1.82 \\
\hline $\begin{array}{l}221 \\
87\end{array}$ & 6.457 & 2 & 2.55 & 2.73 & $\begin{array}{l}223 \\
89\end{array}$ & 6.783 & 2 & 2.60 & 2.51 \\
\hline $\begin{array}{l}224 \\
89\end{array}$ & 6.327 & 1 & 5.73 & 5.31 & $\begin{array}{l}225 \\
89\end{array}$ & 5.935 & 2 & 6.23 & 6.34 \\
\hline $\begin{array}{l}225 \\
91\end{array}$ & 7.390 & 2 & 0.39 & 0.47 & $\begin{array}{l}226 \\
89\end{array}$ & 5.537 & 2 & 9.25 & 9.42 \\
\hline $\begin{array}{l}228 \\
91\end{array}$ & 6.264 & 3 & 7.60 & 7.23 & $\begin{array}{l}229 \\
91\end{array}$ & 5.835 & $1(3)$ & 10.03 & 10.02 \\
\hline $\begin{array}{l}230 \\
91\end{array}$ & 5.439 & 2 & 11.31 & 10.95 & $\begin{array}{l}235 \\
93\end{array}$ & 5.194 & 1 & 13.94 & 13.62 \\
\hline $\begin{array}{l}235 \\
95\end{array}$ & 6.610 & 1 & 5.17 & 5.35 & ${ }_{93}^{237}$ & 4.958 & 1 & 16.19 & 15.75 \\
\hline $\begin{array}{l}239 \\
95\end{array}$ & 5.922 & 1 & 11.11 & 10.92 & ${ }_{95}^{241}$ & 5.638 & 1 & 12.60 & 12.50 \\
\hline $\begin{array}{l}243 \\
95\end{array}$ & 5.439 & 1 & 14.16 & 13.67 & $\begin{array}{l}245 \\
97\end{array}$ & 6.455 & 2 & 9.37 & 9.79 \\
\hline $\begin{array}{l}245 \\
99\end{array}$ & 7.909 & 3 & 3.52 & 3.37 & $\begin{array}{l}249 \\
97\end{array}$ & 5.525 & 2 & 13.61 & 13.55 \\
\hline $\begin{array}{l}252 \\
99\end{array}$ & 6.790 & 1 & 7.83 & 7.85 & $\begin{array}{l}257 \\
101\end{array}$ & 7.558 & $1(4)$ & 7.57 & 7.83 \\
\hline
\end{tabular}




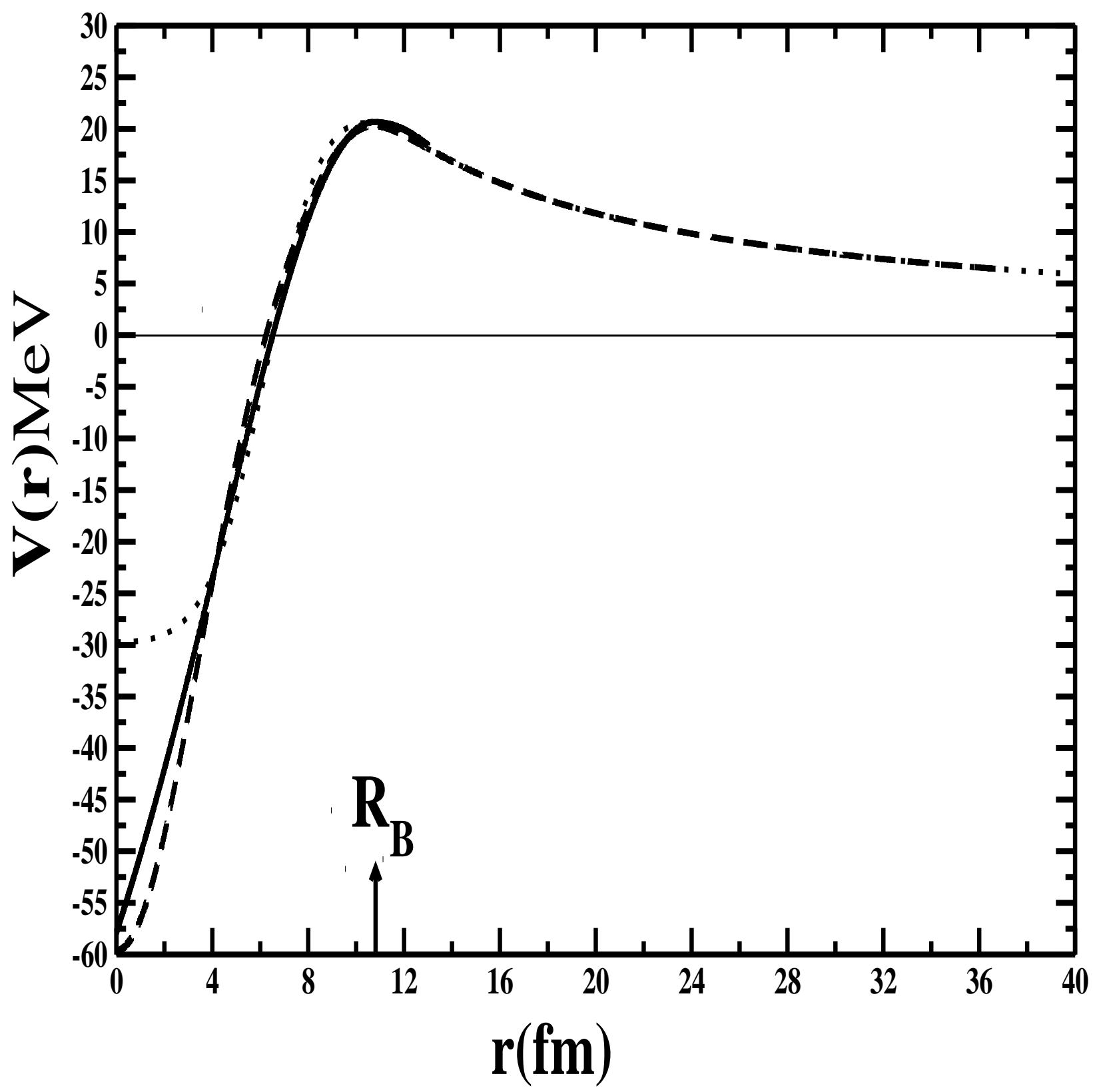

FIG. 1: Plot of potential V(r) as a function of radial distance for the $\alpha+{ }^{208} \mathrm{~Pb}$ system. The dashed curve represents the sum of real nuclear potential expressed by (10) with parameters $\mathrm{V}_{0}=22 \mathrm{MeV}$, $\mathrm{a}_{s}=0.62 \mathrm{fm}, \mathrm{r}_{s}=1.27 \mathrm{fm}, \mathrm{r}_{v}=0.66 \mathrm{fm}, \delta=3.5$ and the Coulomb potential given by (12) with $\mathrm{r}_{C}=1.2$ $\mathrm{fm}$. The solid curve represents the potential expressed by (16) in the text with parameters $\mathrm{r}_{0}=1.07$ $\mathrm{fm}, \mathrm{a}_{g}=0.63 \mathrm{fm}, \mathrm{H}_{0}=1 \mathrm{MeV}, \xi_{1}=-100$, and $\xi_{2}=\mathrm{V}_{B}=20.27$. The arrow indicates barrier position $\mathrm{R}_{B}=10.75 \mathrm{fm}$. The dotted curve represents the potential calculated using RMF theory [18]. 


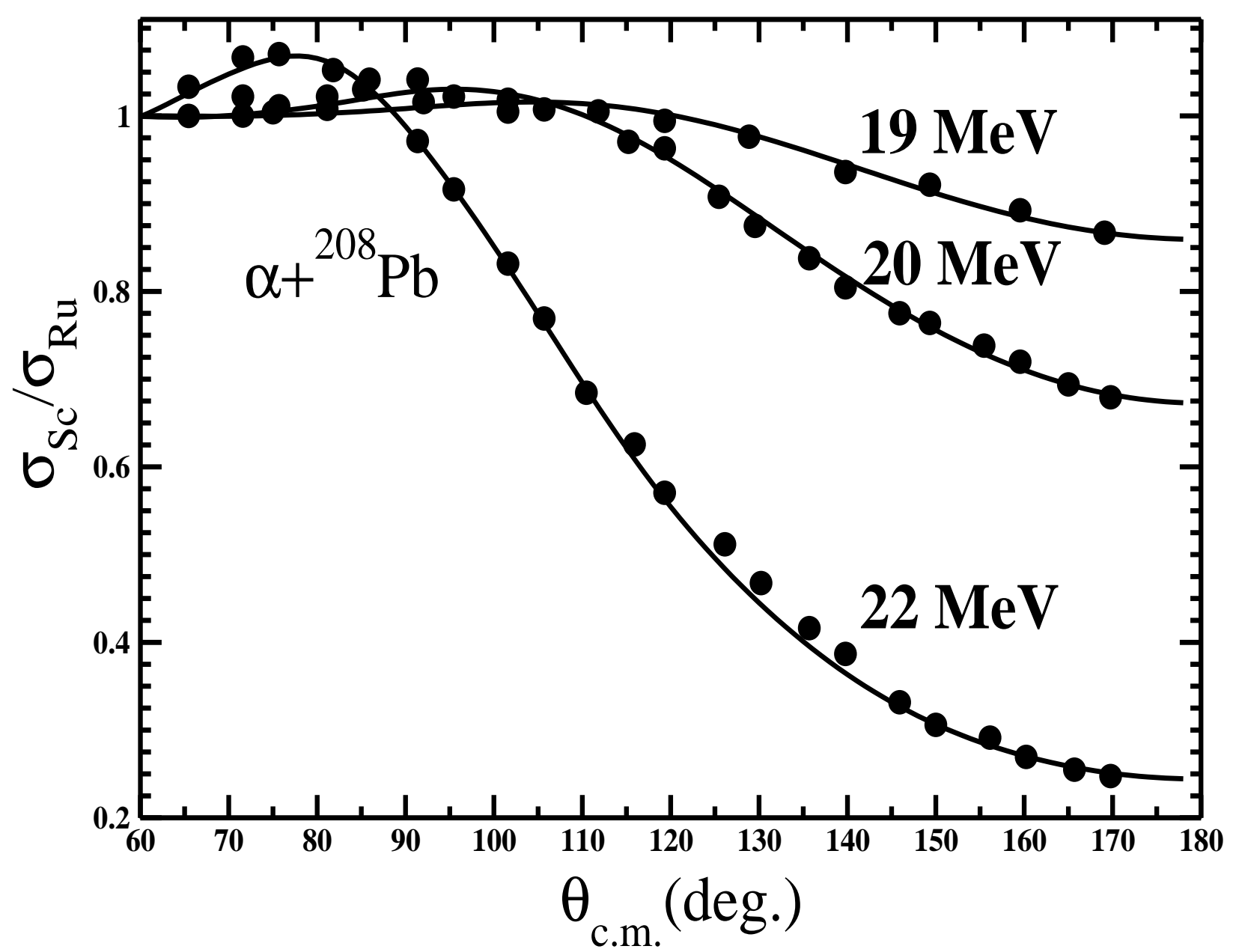

FIG. 2: Angular variation of elastic differential scattering cross section $\sigma_{S c}$, relative to Rutherford $\sigma_{R u}$, of $\alpha$ particles scattered by ${ }^{208} \mathrm{~Pb}$ at 19, 20 and $22 \mathrm{Mev}$ laboratory energies. The solid curves represent the results of present optical model calculations using the parameters: $V_{0}=22 \mathrm{MeV}$, $r_{s}=1.27 \mathrm{fm}, a_{s}=0.62 \mathrm{fm}, r_{v}=0.66 \mathrm{fm}, \delta=3.5, r_{C}=1.2 \mathrm{fm}, W_{0}=5 \mathrm{MeV}, a_{I}=0.28 \mathrm{fm}, r_{I}=1.2 \mathrm{fm}$ for $19 \mathrm{MeV}, r_{I}=1.3 \mathrm{fm}$ for $20 \mathrm{MeV}$, and $r_{I}=1.37 \mathrm{fm}$ for $22 \mathrm{MeV}$. The experimental data shown by solid circles are obtained from [23]. 


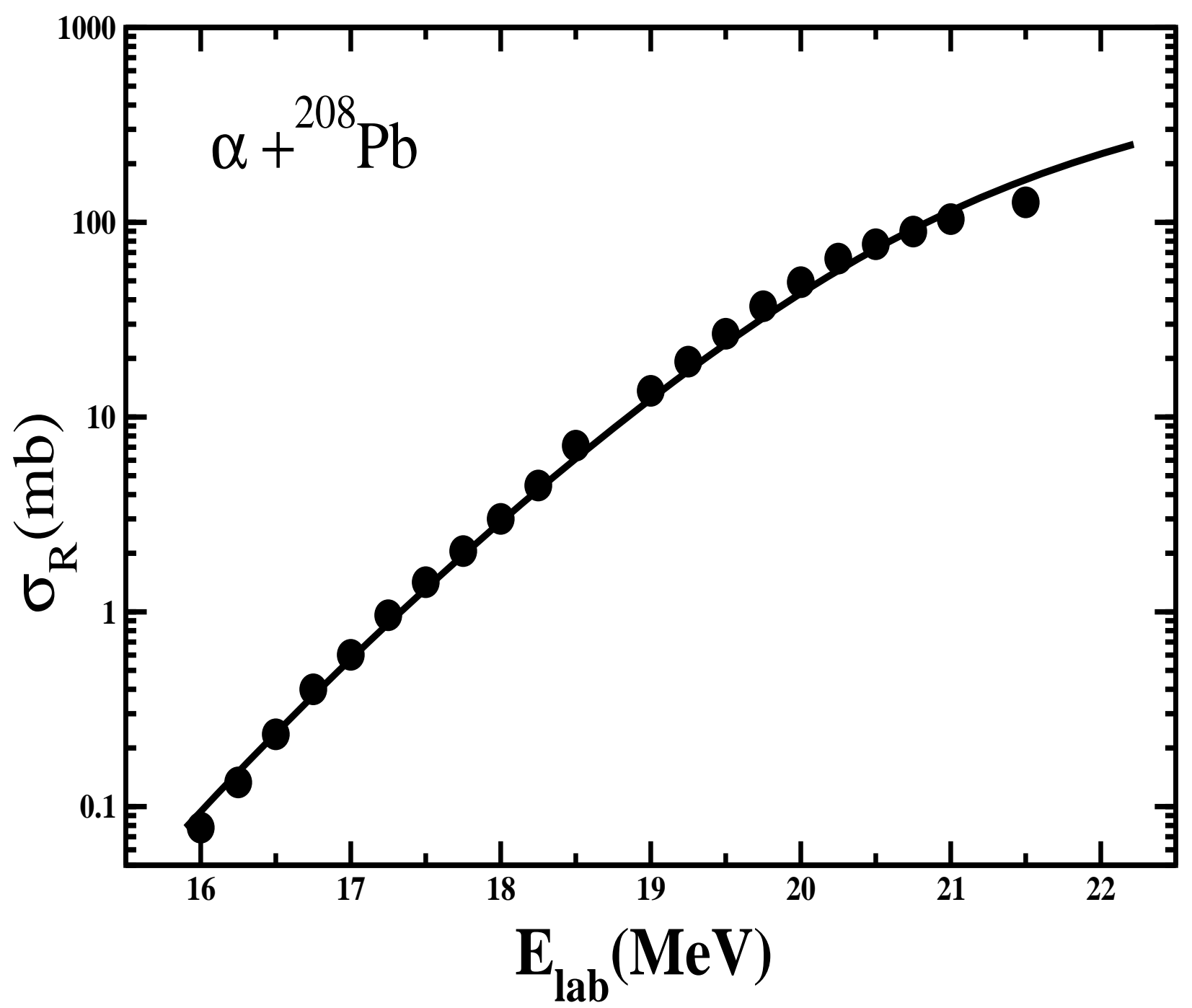

FIG. 3: Plot of reaction cross section $\sigma_{R}$ as function of laboratory energy for the $\alpha+{ }^{208} \mathrm{~Pb}$ collision. The solid curve represents the results of present optical model calculation. The experimental data shown by solid circles are taken from [23]. 